\title{
Interfaces
}

\section{Expérience synesthésique : la partition colorée de «La Valse » de Maurice Ravel}

\section{Gabrielle Thierry}

\section{(2) OpenEdition}

1 Journals

\section{Édition électronique}

URL : http://journals.openedition.org/interfaces/229

DOI : 10.4000/interfaces.229

ISSN : 2647-6754

Éditeur :

Université de Bourgogne, Université de Paris, College of the Holy Cross

\section{Édition imprimée}

Date de publication : 1 janvier 2015

Pagination : 10-27

ISBN : 9780983175247

ISSN : $1164-6225$

\section{Référence électronique}

Gabrielle Thierry, «Expérience synesthésique : la partition colorée de « La Valse » de Maurice Ravel », Interfaces [En ligne], 36 | 2015, mis en ligne le 01 janvier 2018, consulté le 07 janvier 2021. URL : http:// journals.openedition.org/interfaces/229; DOI : https://doi.org/10.4000/interfaces.229

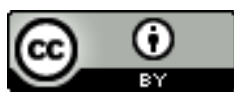

Les contenus de la revue Interfaces sont mis à disposition selon les termes de la Licence Creative Commons Attribution 4.0 International. 


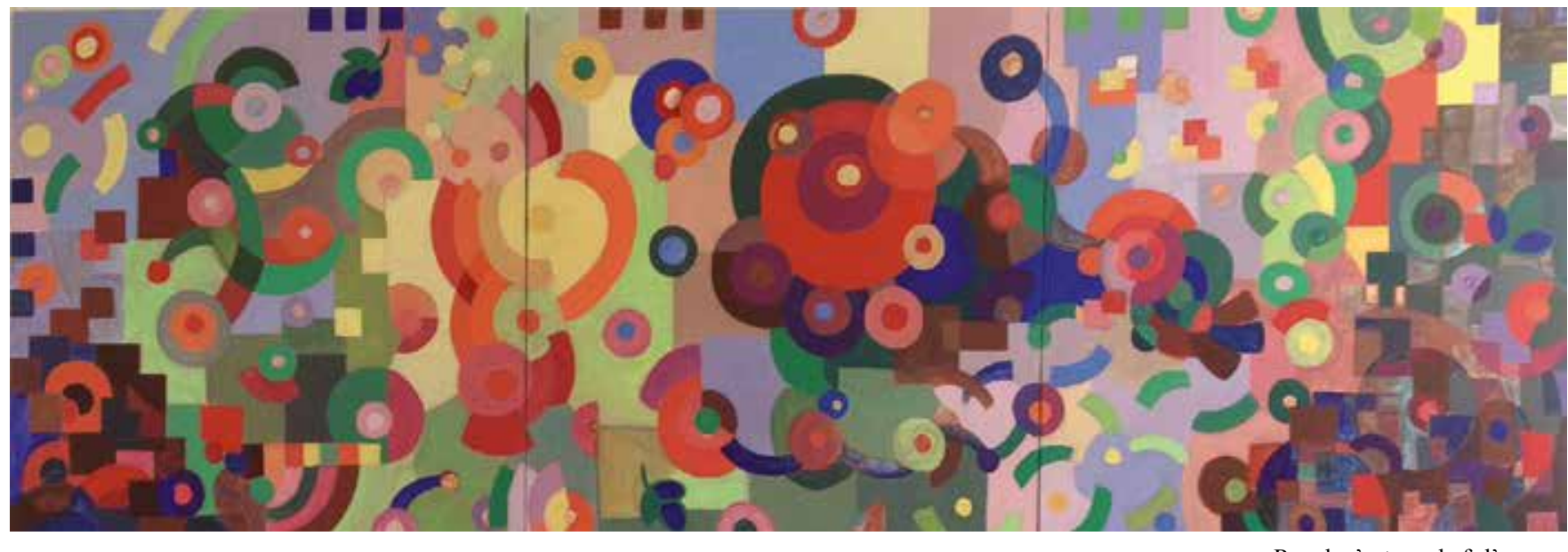

« Ravel, c'est un chef-d'œuvre, mais ce n'est pas un ballet.

C'est la peinture d'un ballet. »

Diaghilev à propos de « La Valse », 1920 


\title{
EXPÉRIENCE SYNESTHÉSIQUE : \\ LA PARTITION COLORÉE DE « LA VALSE » DE MAURICE RAVEL
}

\author{
Gabrielle Thierry
}

Je souhaite avant tout remercier l'Université de Bourgogne, associée au College of the Holy Cross (Worcester, Mass.) et à l'Université Paris-Diderot, pour leur invitation, ainsi que toute l'équipe du Centre Interlangues TIL Texte-Image-Langage. Dans le cadre du colloque sur la Synesthésie organisé à Dijon en juin 2014, j'ai proposé de présenter mon travail en tant qu'artiste peintre musicaliste. Cette démarche personnelle de transposition de la musique sur la toile sera illustrée par « La Valse » de Maurice Ravel. Cette musique a un lien très particulier avec l'histoire. Ravel avait pour ambition d'écrire un hymne aux Valses de Strauss. L'entrée en guerre retarda son projet et eut aussi un impact sur sa création. Dans le cadre du centenaire de la Grande Guerre, cette hymne a une signification toute particulière. « J'ai conçu cette œuvre comme un espèce d'apothéose de la valse viennoise, à laquelle se mêle, dans mon esprit, l'impression d'un tournoiement fantastique et fatal », disait Ravel ${ }^{1}$.

\section{Au commencement : Paysage, musique, peinture}

Pour commencer, je veux rappeler que le paysage comprend, pour moi, une musique qui lui est propre et inhérente. En tant qu'artiste, peindre le paysage, c'est représenter son espace avec ses rythmes et sa musique. La question de la représentation de la musique est riche : s'agit-il d'une représentation de la couleur des sons ou bien d'une retranscription, par le jeu des couleurs et des formes, de l'émotion ressentie à l'écoute de la musique ? Quels liens réels, imaginaires ou émotionnels a-t-elle avec le paysage ? Un paysage peut-il faire l'objet d'une représentation musicale ? Des compositeurs ont été inspirés par le paysage tels Franz Liszt ou Franz Schubert traversant les Alpes, etc. Mais quelle est la part du paysage dans la composition ? En existe-t-il vraiment une forme de transposition?

On sait qu'en écoutant une musique, certaines personnes perçoivent une image, des couleurs, des formes en mouvement. Inversement, peut-on évoquer ou imaginer une musique, un son ou une mélodie à la vue d'un paysage ? Nous serions tous plus ou moins sensibles aux associations de la musique, des couleurs et des formes. Cette capacité, la synesthésie, disparaîtrait rapidement lors de la croissance

1 Maurice RAVEL, «Une Esquisse autobiographique » [dictée à Roland-Manuel, octobre 1928], La Revue musicale, déc.1938, pp. 17-33. 
sauf chez certaines personnes. Il faut noter aussi que selon les époques et selon les écoles, l'évolution des connaissances, l'éducation, les codes et les pratiques culturelles aboutissent à accorder ou à laisser une place plus ou moins importante à cette sensibilité universelle et potentiellement si riche d'expériences esthétiques.

Le processus qui
permet d'exprimer - dans l'image - la musique d'un paysage est le fruit d'une recherche esthétique et artistique. Reproduire visuellement le rythme des paysages est une

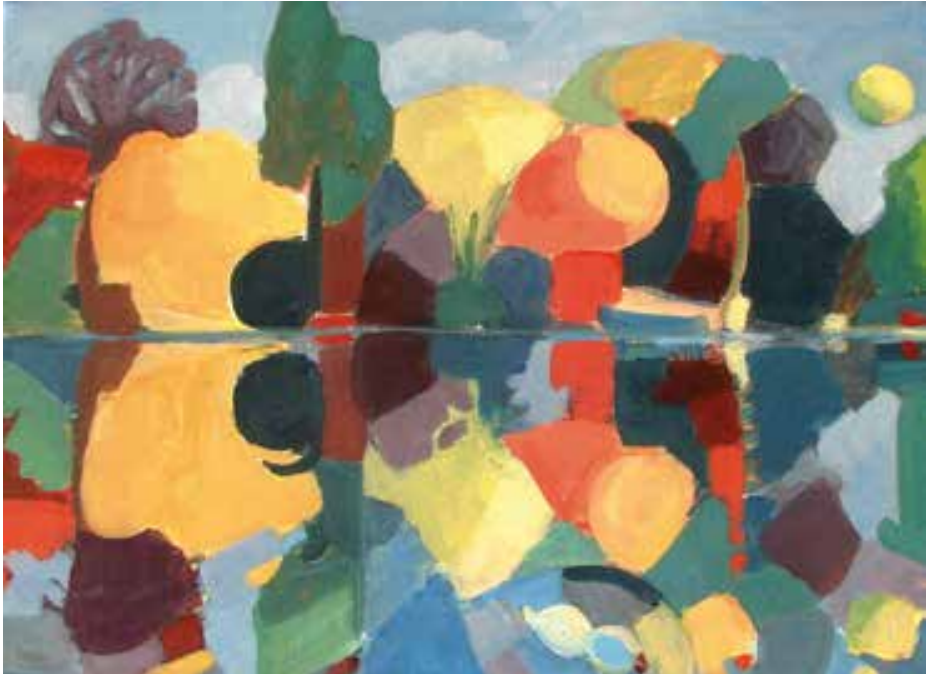

Illustration $1:$ «Andrésy Flamboyant »,

Huile sur Toile première étape sans doute plus évidente, notamment grâce à la répétition des formes et des aplats de couleurs.

Dans le tableau « Andrésy flamboyant » (Ill. 1), les couleurs d'un début d'automne sur les berges de la Seine ${ }^{2}$ et les rythmes sont naturellement présents dans les reflets. L'émotion musicale est issue de la lumière et de ces couleurs vives. La couleur me semble alors être une "donnée musicale » qui s'impose presque physiologiquement.

Ce tableau, qui a pu servir de révélateur, a motivé la poursuite de ma recherche sur la représentation de la musique des paysages. Par la suite, la série de tableaux intitulés «Partitions

2 Ce tableau a été réalisé un peu à la manière du « Talisman » de Paul Sérusier, tableau peint sous les directives de Gauguin, dont l'objectif était de conjurer une magie des couleurs et des lignes indépendante de toute représentation : «Comment voyez-vous cet arbre ? », avait dit Gauguin à Sérusier devant un coin du Bois d'Amour. « Il est bien vert ? Mettez donc du vert, le plus beau vert de votre palette. Et cette ombre, plutôt bleue ? Ne craignez pas de la peindre aussi bleue que possible ». Ainsi nous fut présenté pour la première fois (1888), sous une forme paradoxale et inoubliable, le fertile concept de la « surface plane recouverte de couleurs en un certain ordre assemblées ». Ainsi nous connûmes que toute œuvre d'art est une transposition, une caricature, l'équivalent passionné d'une sensation reçue. (Maurice Denis cité Jean-Pierre Guillerm dans Des Mots et des couleurs, p. 197). 
colorées » a permis de définir un vocabulaire pictural de l'espace musical. Ce « vocabulaire » est avant tout fondé sur l'émotion et non pas sur un mécanisme de correspondances directes comme dans la synesthésie " pure ». La recherche des couleurs et des formes est guidée avant tout par une émotion telle que celle que l'on peut ressentir en écoutant de la musique. L'émotion est le ciment de la correspondance paysage-musique / images-sons.

La structure et la composition de la musique jouent un rôle tout aussi primordial dans la composition du tableau. La question de la temporalité est alors prégnante. La représentation picturale des notes dans l'espace peut par exemple prendre la forme de cercles chromatiques qui représentent les ondes sonores. Les couleurs varient selon leur propagation sur les fonds colorés/sonores.

Ainsi, la série de tableaux intitulée «Variations » rassemble des œuvres présentant des paysages et leur musique.

L'ensemble des « Matinales » (Ill. 2), par exemple, consiste en une séquence de paysages représentant les rives de Seine sous la neige. Dans le premier tableau de cette série, les éléments restent figuratifs. Deux autres tableaux, plus petits, sont les témoins d'une recherche des formes existantes dans ce paysage. Une synthétisation qui pourrait être un début de langage des formes et de leur composition. Le mouvement des oies bernaches sur les rives est essentiel pour faire naître une mélodie, celle du violon dans la sonate de J.-S.Bach. La dernière composition aux trois cercles bleus est une nouvelle étape qui met en équilibre sur la toile les éléments musicaux de la sonate, à l'image du balancement des oies. Les notes de piano structurent par leurs éléments rectangulaires l'espace de la toile. C'est une émotion commune qui m'impose ces différentes interprétations colorées, ma démarche s'appuyant sur une abstraction progressive ${ }^{3}$.

Ce qui nous intéresse ici c'est d'utiliser ce vocabulaire pictural de la musique pour transposer une œuvre sonore sur la toile à l'instar de tout langage pour décrire une œuvre, avec ses pertinences sensorielles et émotionnelles.

Ce propos va être illustré par la présentation du tableau « La Valse ».

3 Quelques tableaux de la série Variations sont à découvrir en musique sur le site www.mgbook.com, onglet «Peinture »: « Les Matinales » dans lequel les oies bernaches parcourent les rives de Seine sur une sonate de J.S. Bach, ou « Le Parc» sur le quatuor d'Anton Bruckner (publié dans Florence Collin, ed., « Musique et Arts plastiques : Hommage offert à Michèle Barbe par ses étudiants et amis, Paris, Observatoire musical français, série « Musique et arts plastiques », $\left.\mathrm{n}^{\circ} 7,2011\right)$. 

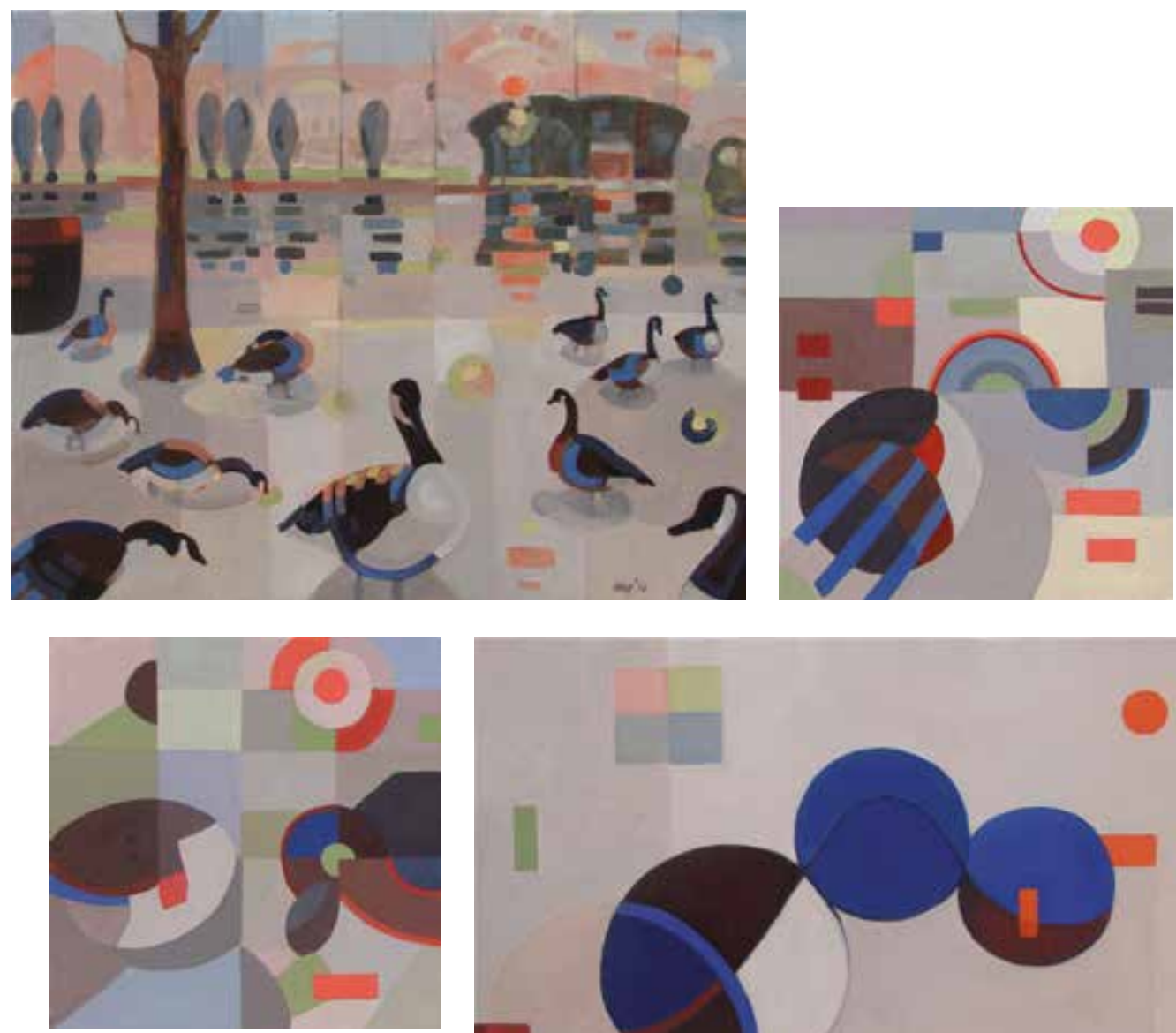

Illustration $2:$ « Les Matinales »

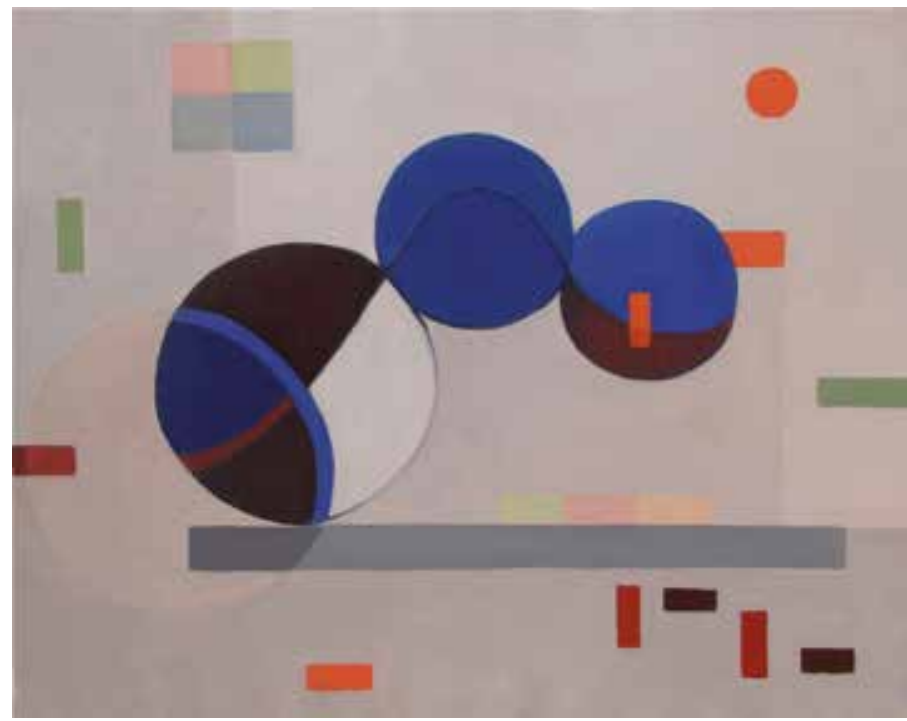

Huile sur toiles en polyptique 


\section{La Valse}

Le tableau, composé de 3 toiles de $100 \times 100 \mathrm{~cm}$, a été peint à la peinture à l'huile, entre janvier et avril 2013. «La Valse » de Ravel a été jouée par l'Orchestre Philarmonique de Radio France, dirigé par Myung-Whun Chung, salle Pleyel ${ }^{4}$. Il s'agit d'un « poème chorégraphique » de 12 minutes.

L'écoute d'une telle musique orchestrale dans un tel lieu a accentué pour moi la dimension matérielle et structurelle de l'œuvre de Ravel. L'harmonie des sons, sa composition toute entière s'apparente à une construction, dense et colorée. On peut faire référence à une architecture et c'est dans ce contexte que la densité de la peinture à l'huile intervient : l'épaisseur des couches, la matité des aplats de couleurs ou sa résonnance dans les glacis offrent autant d'outils pour aiguiser le son, trouver sa juste présence. Il s'agit de traiter non seulement couleurs et formes mais aussi de représenter l'architecture solide de l'œuvre. La représentation doit également rendre compte de la poésie et de la violence exprimée dans l'œuvre par Maurice Ravel. « Ravel a souhaité une synthèse et une apothéose de la Valse Viennoise. Il ne faut pas jouer cette valse en une série d'impressions mais comme une impression totale. Quelques formes y apparaissent comme surgies du passé » explique le chef d'orchestre suisse Ernest Ansermet lors d'une émission télévisée consacrée à « La Valse »5.

La difficulté majeure dans cette entreprise fut de concevoir la représentation de l'ensemble des instruments. Il s'agissait de ressentir et d'écouter un orchestre pour en appréhender l'espace, la couleur et de parvenir à dompter les formes qui s'en échappent. Synesthètes, nous percevons la couleur des sons de différentes manières. Je propose ici la couleur que m'évoque chacun de ces instruments de l'orchestre (Ill. 3 : La couleur des instruments de l'orchestre). Cette composante synesthète est assez bien reconnue en peinture (peut-être plus qu'en musique, sauf rares exceptions comme Messiaen notamment). Le sujet pourrait donner lieu à des recherches bien plus expérimentales et approfondies. Il me semble que là sont données les clés de compréhension de la composition colorée : le son des violons vert, de la harpe mauve, des vents jaunes, des cuivres rouges, des violoncelles et des basses bruns, etc., représente ici la composante « synesthésique » vraie de l'approche proposée.

Dans son ouvrage Le Peintre des sons. Sons et Couleurs, ${ }^{6}$ Charles Blanc-Gatti, peintre des sons et membre fondateur du mouvement musicaliste, entreprend de croiser les regards et analyses des

$4 \quad$ Un extrait du concert est disponible sur internet : http://www.youtube.com/watch?v=TMSgWhIENSk.

5 «Ernest Ansermet et l'OSR: La valse de Ravel » (30 janvier 1958) http://www.rts.ch/video/emissions/ archives/50/3428043-ernest-ansermet-et-1-osr-la-valse-de-ravel.html

6 Charles Blanc-Gatti, Le Peintre des Sons. Sons et Couleurs, Paris, Victor Attinger, 1934. 

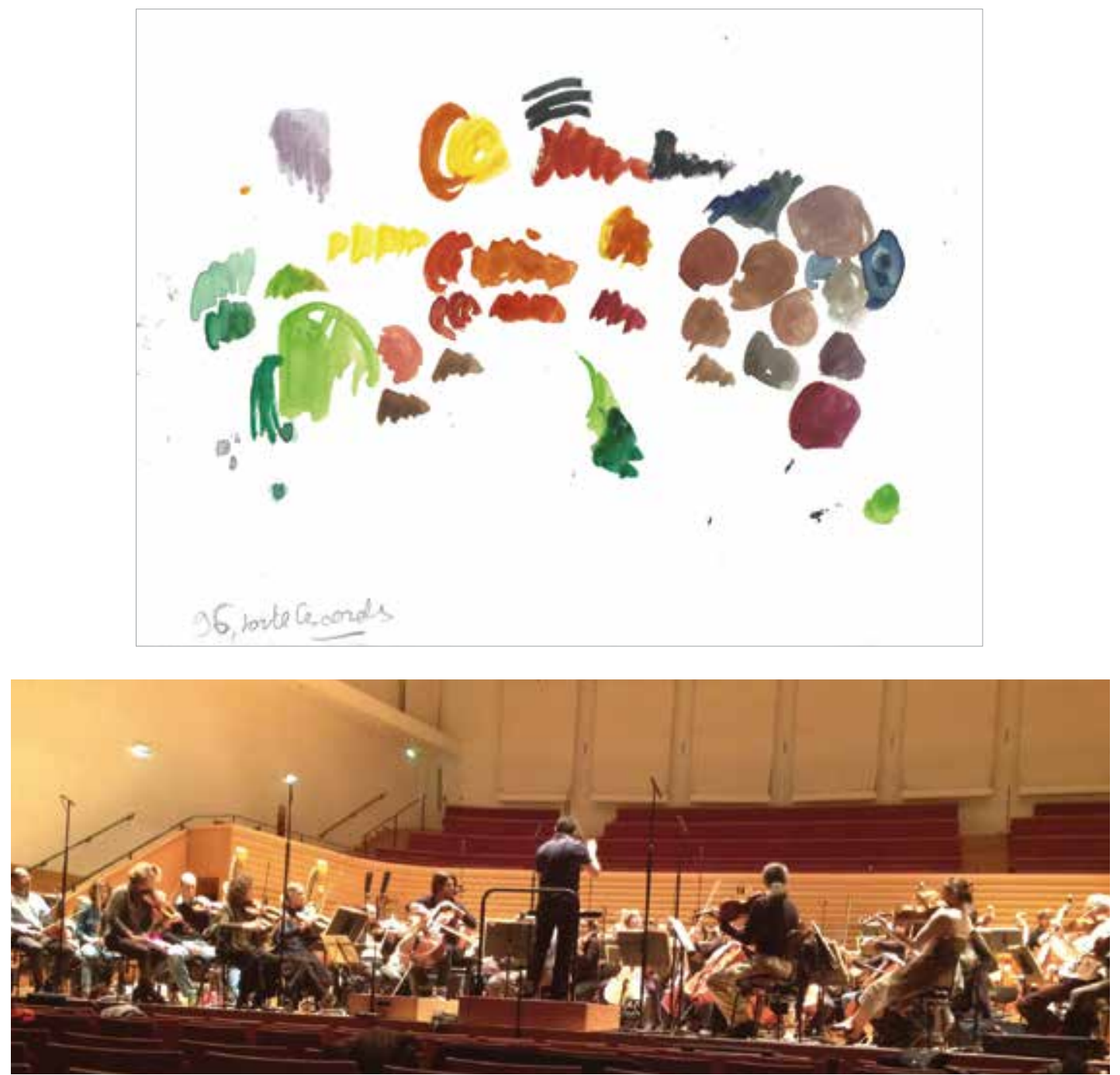

Illustration 3 : Les couleurs de l'orchestre 
peintres, musiciens, poètes, philosophes, scientifiques qui se sont penchés sur l'interaction des arts, et en particulier sur la couleur de la musique. Parmi ces peintres, citons l'américaine Louise Janin, aussi théoricienne de l'esthétique ; le tchèque, Franck Kupka, virtuose des formes abstraites et des harmonies colorées, un des créateurs de l'orphisme avec Robert Delaunay ${ }^{7}$; Paul Klee et sa représentation plastique d'une notation musicale qui donna lieu à une nouvelle représentation de l'écriture musicale. Bien d'autres artistes, tous mouvements confondus, ont fait de la musique un élément indissociable de l'abstraction en peinture : August Macke constate que « [c]e qui rend la musique si mystérieusement belle agit aussi de manière ensorcelante en peinture » ; Morgan Russell veut « créer une pure succession abstraite de formes faites de couleurs qui se meuvent dans un tempo donné avec le son, comme les êtres d'un monde plus plastique, dansant "; avec son piano magique, Vladimir B.-Rossiné projetait dans l'espace ou sur un écran des couleurs et des formes mouvantes et variées à l'infini, qui dépendaient absolument, comme dans le piano sonore, du fonctionnement des touches, bien d'autres suivirent comme Hans qui en explora les possibilités dans le film abstrait ${ }^{8}$.

Un des précurseurs de cette représentation de la musique est Vassily Kandinsky, dont les enseignements au Bauhaus furent les fondations théoriques de l'art total ou peinture et musique avaient leur composante. Relire dans Du spirituel dans l'art est à ce titre tout à fait éclairant. Toute perception synesthète de la musique est avant tout personnelle. Il semblerait que nous ne percevons pas tous la musique de la même manière. Certains vont percevoir surtout la couleur des notes; d'autres vont voir des formes ou encore des images colorées en mouvement, etc. Pour Kandinsky, « musicalement, le bleu clair s'apparente à la flûte ; le foncé au violoncelle, s'il fonce encore à la sonorité somptueuse de la contrebasse ; dans ses tons les plus profonds, les plus majestueux, le bleu est comparable aux sons graves de l'orgue ${ }^{9} »$. Ainsi égraine-t-il sa sensation musicale des couleurs, instrument par instrument

7 L'Orphisme de Robert Delaunay n'est pas centré sur la représentation de la musique. Ses cercles chromatiques avaient pour « but » de proposer un parcours du regard sur la surface de la toile : « L'œil frappé par une couleur, survole la surface de la toile à la recherche de la complémentaire. » Robert Delaunay semblait rechercher le mouvement de la scène et offrait ainsi un parcours du paysage représenté. Même si Delaunay parle en 1912 de « recherche de transparence des couleurs, comparables aux notes musicales », cela ne l'empêche nullement de revendiquer la spécificité rétinienne de la peinture fondée sur des "lois optiques » propres, indépendantes des accords musicaux. Il introduit néanmoins la question des rythmes dans l'espace de la représentation. (Pascal Rousseau, J.P. Ameline, eds, Robert Delaunay 1906-1914 : De l'impressionnisme à l'abstraction, Paris, Editions du Centre Pompidou, 1999, p. 87).

8 Marcella Lista, Sophie Duplaix, eds, Sons et Lumière : Une histoire du son dans l'art du XXème siècle, Paris, Editions du Centre Pompidou, 2004, respectivement p. 21 - 136 - 148 et 158.

9 Vassily Kandinsky, Du spirituel dans l'art et dans la peinture en particulier, Paris, Gallimard (Folio-Essais), 1996, p. 150. 
tout en exprimant la difficulté de l'expliquer avec des mots. « Les tons des couleurs, comme ceux de la musique, sont d'une nature beaucoup plus fine, provoquant dans l'âme des vibrations beaucoup plus subtiles, indescriptible par les mots ${ }^{10} »$. Pour ma part, je ne vois pas les couleurs, je recherche sur la palette la couleur qui me procure la même émotion que tel ou tel instrument. La complexité de « La Valse » de Ravel est dans sa composition, dans l'imbrication des différentes parties musicales que l'œuvre offre en répétition tout au long de ses 12 minutes d'intensité. La toile va chercher à représenter cette complexité et à la valoriser, pour le plaisir des sens mêlés.

Les premières esquisses de composition permettent déjà une appropriation de l'œuvre (Ill. 4). Alors que j'exécute la structure de l'œuvre en écoutant « La Valse » (Ill. 6), je cherche les formes exactes, les liaisons et les transitions du jeu de chaque groupe d'instruments dans cette pièce musicale complexe (Ill. 7). La musique est représentée dans un espace. Les notes évoluent ici sur un fond sonore lié aux instruments en fonction de leur présence sur différents plans qui peuvent être évoqués. Ainsi, l'instrument qui donne la ligne mélodique apparaît au premier plan. La colorisation est réalisée en couches successives. Les verts sont attribués aux violons (Ill. 8), puis tous les instruments sont joués/ peints en couleurs au moment même où ils sont joués, sur une forme nouvelle de portée musicale.

Le fait que certains passages soient joués et rejoués lors des répétitions de l'orchestre, avec des modulations dans le rythme, l'harmonie et l'intensité — des modulations demandées par le chef d'orchestre - m'a confortée dans l'idée que musique et peinture sont faites de la même matière. Je propose de travailler la peinture sur ma toile comme un musicien assure la justesse de la sonorité de son instrument. Je cherche les rythmes, les formes sonores, leurs imbrications. Ainsi, les ondes de propagation peuvent être représentées sous la forme de cercles concentriques colorés tandis que les fonds sonores construisent un «passé musical ». Cette distinction permet de créer la temporalité qui sera aussi bien celle du regard que celle de l'audition musicale. L'évolution de la note dans son espace, sa représentation, peut ainsi être associée à son mouvement dans l'espace musical. Ce choix explique que la transposition picturale de « La Valse » reproduit le mouvement de lecture linéaire, de gauche à droite, et mime la temporalité.

Citons Olivier Messiaen qui accordait une grande importance aux formes de relations entre musique et couleurs : " Je me suis rendu compte que, moi aussi, je liais les couleurs aux sons, mais intellectuellement, pas par les yeux. En effet, depuis toujours, lorsque j'entends ou lorsque je lis de la musique (en l'entendant intérieurement), je vois dans ma tête des complexes de couleurs qui marchent et bougent avec les complexes des sons. À force d'observer ce qui se passait en moi, j'en ai déduit une loi ${ }^{11} \gg$.

$10 \quad$ Ibid., p. 164.

11 Claude Samuel, Anik Lesure, eds, Olivier Messiaen : Le Livre du Centenaire, Paris, Symétrie, 2008, p. 147. 

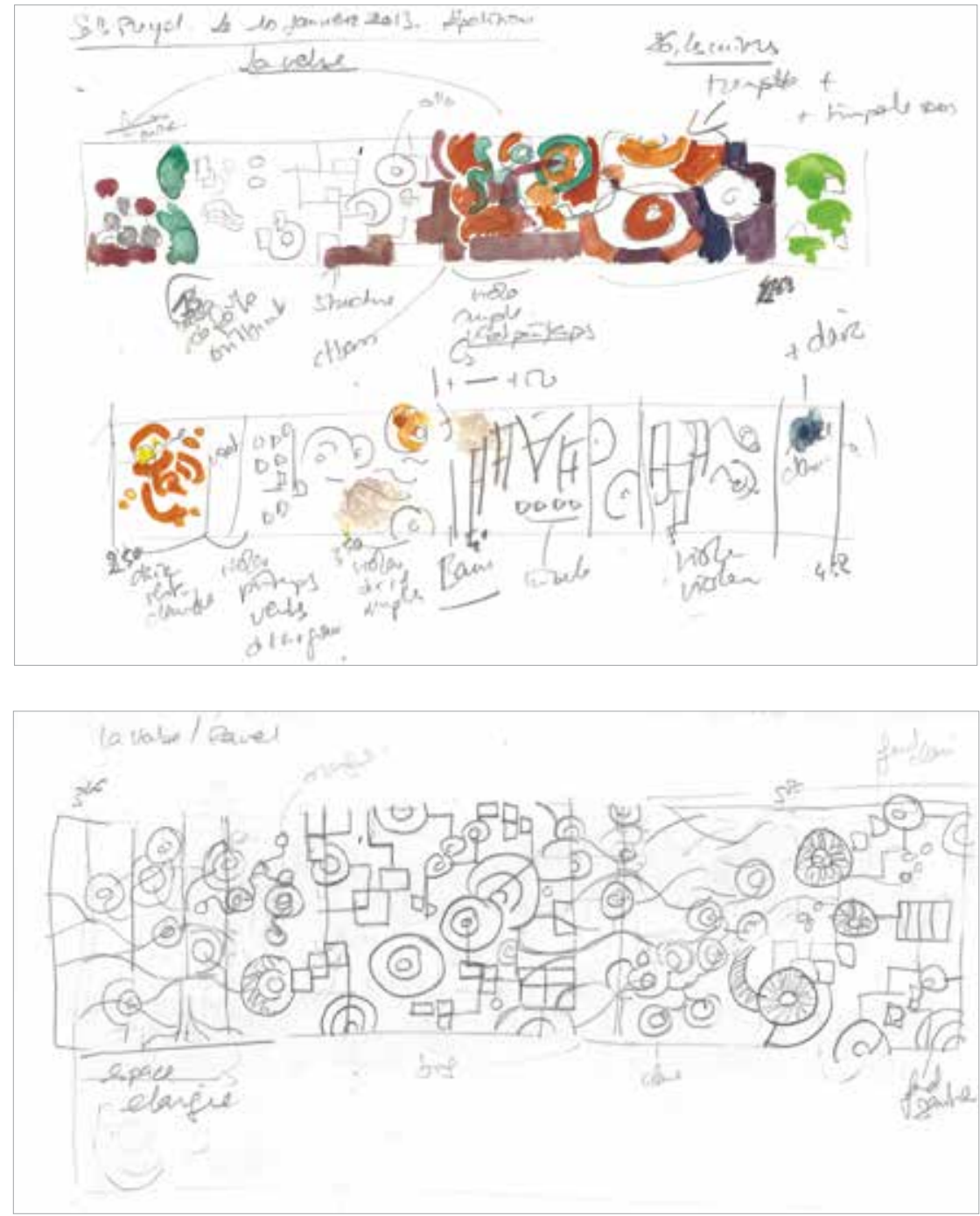

Illustration 4 : tentatives de composition 
Les ébauches aquarellées permettent de travailler aussi bien la composition et la structure que les couleurs, trouvées lors de l'audition pendant les répétitions à la Salle Pleyel.

Le travail définitif préalable à la réalisation de la peinture repose sur la réalisation d'une dernière aquarelle en atelier (Ill. 5). Celle-ci, telle une partition, représente une phrase musicale qui va être jouée plusieurs fois. Plus on avance dans le temps, plus cette phrase musicale intègre intensité et tension du jeu musical. La tension va croître jusqu'au drame final, apocalyptique qui signe la mort de la valse évoquant une suffocation ultime.

Une fois effectuées ces multiples ébauches de couleurs et de compositions formelles, la version définitive à l'huile de « La Valse » est réalisée sur un grand format $(100 \times 300 \mathrm{~cm})$. Sa construction résulte d'un travail visant à ce que chaque extrait de musique ait sa place dans le tableau : la valse jouée par les violons, sa correspondance avec les vents, puis avec les cuivres en fracas, puis la montée stridente des violons pour aboutir à la suffocation finale assourdissante. Le tableau décrit un espace, dont les dimensions offrent à percevoir une temporalité. Je me situe dans l'espace que le compositeur a créé et je m'efforce de le retranscrire sur la toile.

Pour évoquer notre immersion physique dans la musique, je figure parfois des feuilles qui volent. Elles évoquent notre présence, et le fait que nous «tournoyons » dans la musique lorsqu'elle libère nos émotions. Ici, trois feuilles dansent sur cette valse à trois temps " imaginée » et « mise en image » en triptyque. De la même façon qu'un compositeur peut livrer, sur la partition et parfois dans des textes, des clés de lecture et d'interprétation, le peintre peut aussi bien expliciter son œuvre.

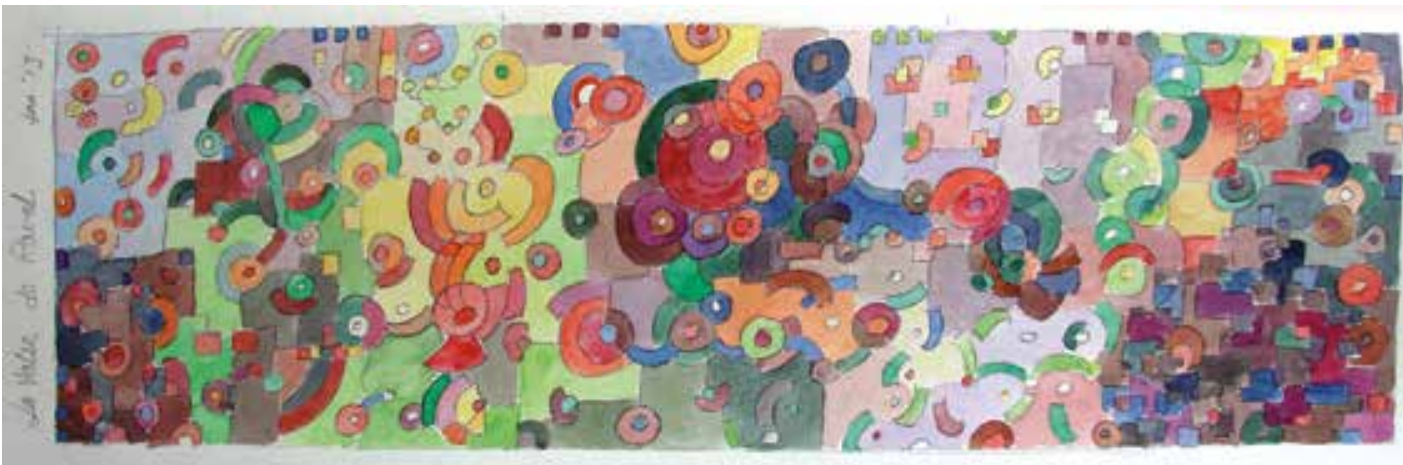

Illustration 5 : première aquarelle de la Valse 

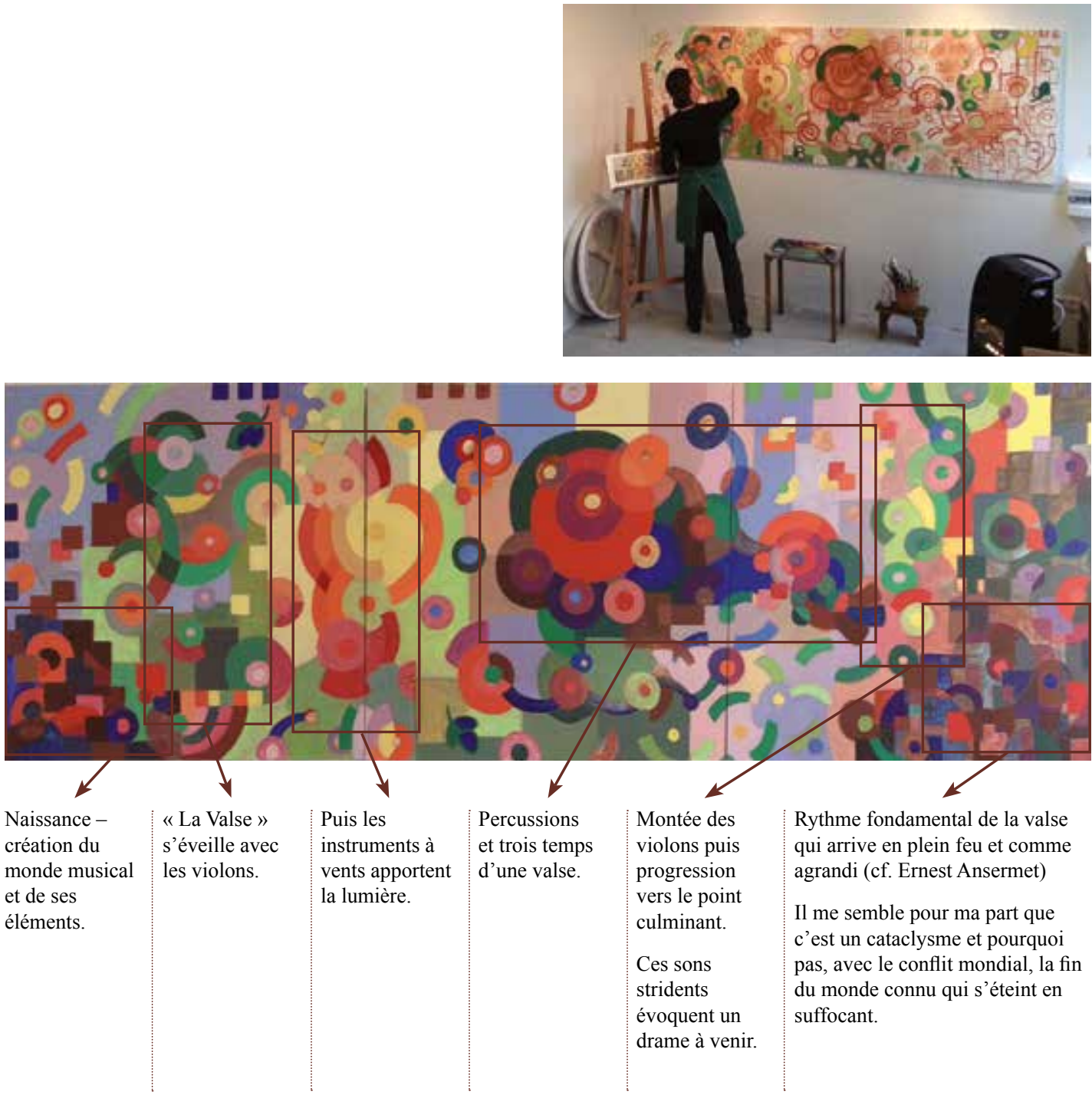


\section{Pour conclure}

Le paysage comme point de départ a permis de retranscrire un espace musical de correspondances à partir d'une perception particulière faisant appel au "sentiment synesthésique ». La transposition de la musique en peinture est une recherche esthétique constante et la présentation de « La Valse » de Maurice Ravel en est une tentative de démonstration. L'invention d'un « vocabulaire pictural » de la musique m'a permis une représentation par l'image. Les formes de recomposition entre musique et peinture sont avant tout inspirées par le ressenti d'émotions et engendrées par la recherche de correspondances. De l'image pourrait naître une émotion rythmique et colorée de la musique, une émotion toute musicale. Cette fusion n'est pas nouvelle, son importance est cependant relativement méconnue, notamment pour son rôle dans l'émergence de l'abstraction en peinture. Il me semble que nous pourrions reconsidérer aujourd'hui la relation entre musique et peinture ou musique et représentation picturale. Voir exposée La Valse au-dessus de l'orchestre lors d'un concert serait l'aboutissement de ce projet. Il permettrait d'offrir au public cette interprétation picturale de la musique, une émotion particulière qui va dans le sens de l'histoire des arts. « On en viendra un jour à exécuter des symphonies en même temps qu'on représentera de beaux tableaux, pour en augmenter l'impression », écrivait Eugène Delacroix dans son Journal (9 avril 1856) ${ }^{12}$.

12 Eugène Delacroix cité par Jean d'Elbée dans « Le Sourd et le muet (Notes parallèles sur Goya et Delacroix) », La Revue hebdomadaire, Paris, Plon, Vol. 30, 1930, p. 452. 


\section{Ouvrages Cités}

BLANC-GATTI, Le Peintre des Sons. Sons et Couleurs, Paris, Victor Attinger, 1934.

COLLIN, Florence, ed., Musique et Arts plastiques : Hommage offert à Michèle Barbe par ses étudiants et amis, Paris, Observatoire musical français, série « Musique et arts plastiques », $\mathrm{n}^{\circ} 7,2011$.

D’ELBÉE, Jean, « Le Sourd et le muet (Notes parallèles sur Goya et Delacroix) », La Revue hebdomadaire, Paris, Plon, Vol. 30, 1930, pp. 437-64.

GUILLERM, Jean-Pierre, ed., Des Mots et des couleOurs, Lille, Presses Universitaires du Septentrion, 1979.

KANDINSKY, Vassily, Du spirituel dans l'art et dans la peinture en particulier, Paris, Gallimard (Folio-Essais), 1996.

LARNER, Gerald, Maurice Ravel, London, Phaidon, 1996.

LISTA Marcella, DUPLAIX Sophie, eds, Sons et Lumière : Une histoire du son dans l'art du XXème siècle, Paris, Editions du Centre Pompidou, 2004.

RAVEL, Maurice. «Une Esquisse autobiographique » [dictée à Roland-Manuel, octobre 1928], La Revue musicale, déc. 1938.

ROUSSEAU, Pascal, AMELINE, J.P., eds, Robert Delaunay 1906-1914 : De l'impressionnisme à l'abstraction, Paris, Editions du Centre Pompidou, 1999.

SAMUEL, Claude, LESURE, Anik, eds Olivier Messiaen : Le Livre du Centenaire, Paris, Symétrie, 2008. 


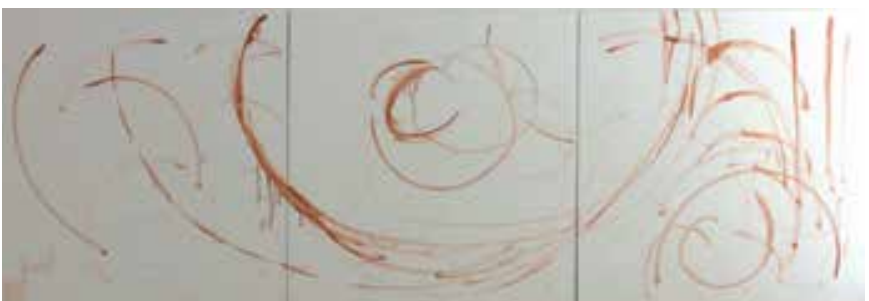

Illustration 6 : charpente sous-jacente, le geste

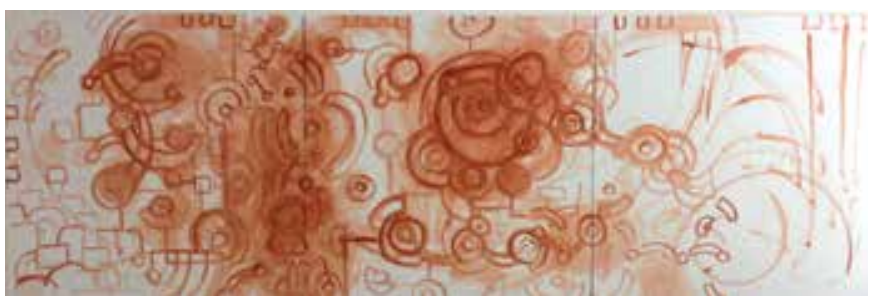

Illustration 7 : composition du tableau

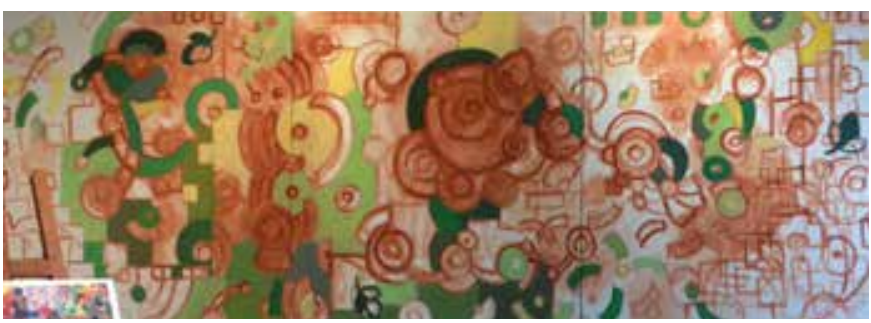

Illustration $8:$ premières couleurs, les violons

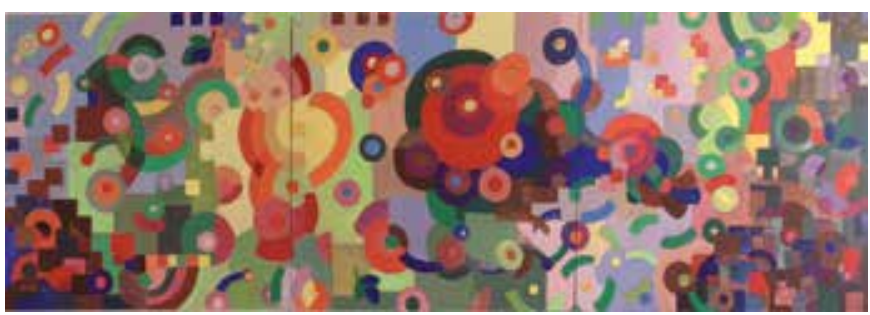

Illustration 9 : harmonie des sons/couleurs 


\section{Deux Encadrés}

\section{Petite histoire de « La Valse »}

En accord avec Serge de Diaghilev, Maurice Ravel envisage dès 1906 de composer pour le ballet une Apothéose de la valse en hommage à Johann Strauss, mais la Première Guerre mondiale l'oblige à remettre ses projets. Vécue comme un anéantissement de la civilisation, celle-ci vient en effet changer la donne. À l'image romantique et fastueuse de la cour viennoise du XIXe siècle, si bien illustrée par les Valses de Johann Strauss II, succède l'image d'un monde décadent menacé par la barbarie.

Pour cette raison l'œuvre de Ravel dépasse de très loin ses ambitions initiales. Le musicien compose selon sa propre expression un « tourbillon fantastique et fatal », somptueuse évocation de la grandeur, de la décadence puis de la destruction de la civilisation occidentale.

« La Valse » est présentée à Diaghilev en avril 1920 dans les appartements de Misia, en présence de nombreux amis musiciens, chorégraphes, écrivains etc. Francis Poulenc relate ainsi l'évènement : « Diaghilev devait monter la Valse aux Ballets Russes (...). Ravel est arrivé très simplement avec sa musique sous son bras et Diaghilev lui dit : " Eh bien, mon cher Ravel, quel bonheur d'entendre la Valse... ». Et moi qui connaissais très bien à cette époque Diaghilev, j'avais vu le râtelier bouger, le monocle bouger, j'avais vu qu'il était embarrassé, qu'il n'aimait pas cela, qu'il allait dire : « Non. » Quand Ravel eut terminé, Diaghilev lui a dit un mot que je crois très juste. Il a dit «Ravel, c'est un chef-d'œuvre, mais ce n'est pas un ballet. C'est la peinture d'un ballet. » Mais ce qu'il y a eu d'extraordinaire, c'est que Stravinsky n'a pas dit un mot, Rien! Alors, moi, c'était en 1921, j'avais vingt-deux ans, j'étais sidéré et ça m'a donné pour la vie entière, une leçon de modestie car Ravel a repris sa musique tout tranquillement, sans se soucier de ce qu'on pouvait en penser, et il est reparti, bien calmement. »

Gerald Larner, Maurice Ravel, London, Phaidon, 1996, pp. 172-3. Cet événement est également relaté par Francis Poulenc dans Moi et mes amis (Paris, La Palatine, 1963). 


\section{Les partitions colorées des Nymphéas}
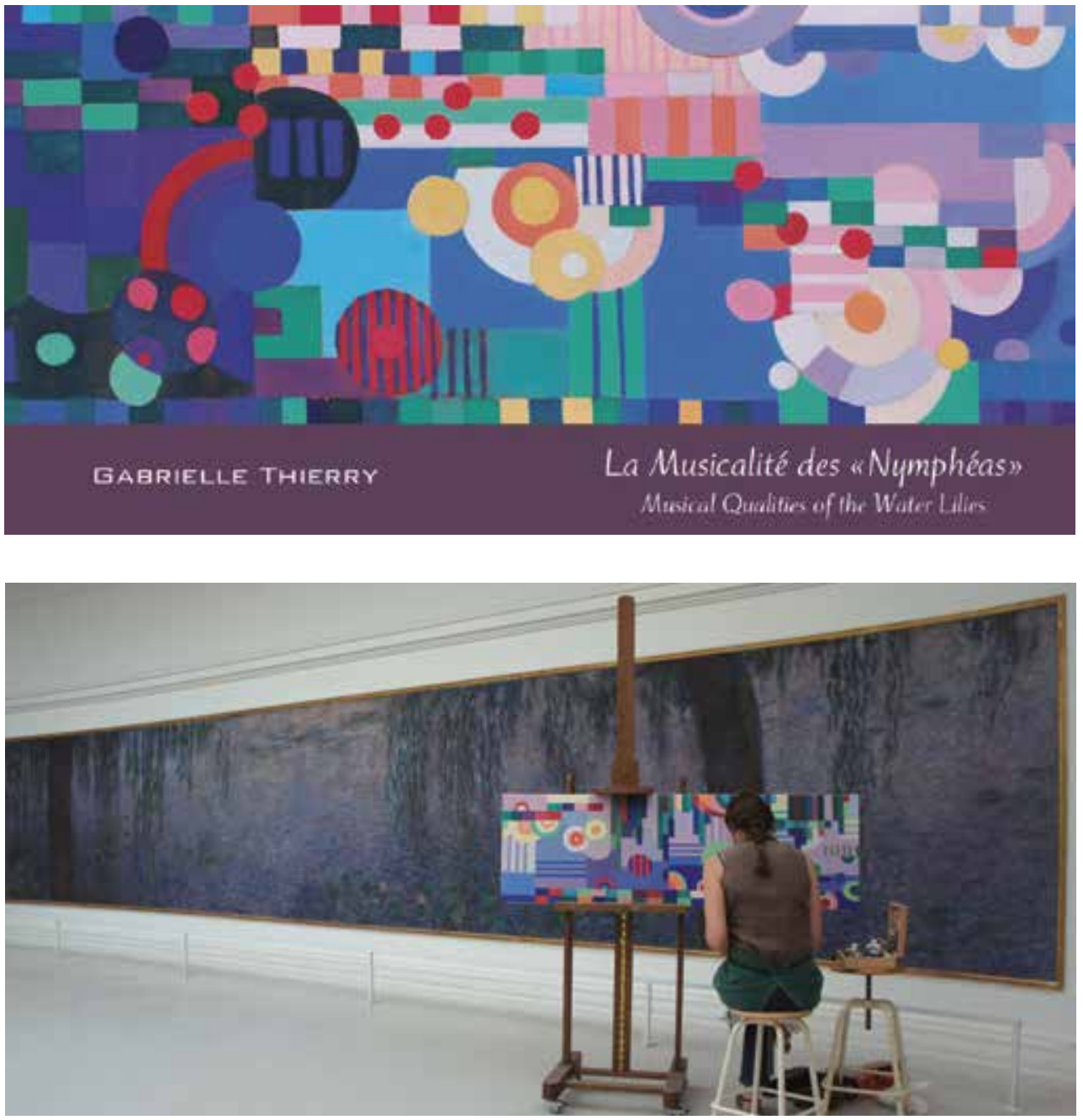


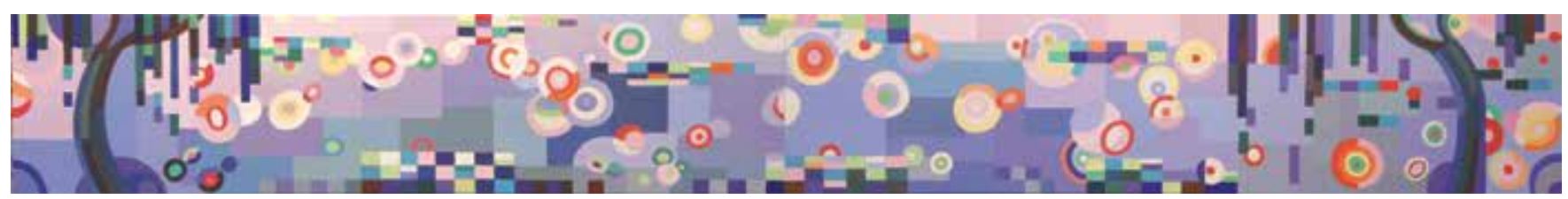

«Musicalité des Nymphéas, Les Deux Saules », 2011, Huile sur toile, en diptyque, 50x 424 cm

Parmi les expériences picturales que j'ai menées pour mettre en évidence la relation entre paysage et musique, on peut citer une série intitulée « Les Partitions colorées des Nymphéas de Claude Monet ». Cette sensation musicale m'est apparue évidente lors d'une visite du Musée de l'Orangerie en 2010.

Avec une autorisation spéciale, j'ai pu peindre pendant 18 mois devant les paysages d'eau de Claude Monet exposés au Musée de l'Orangerie. Les quelques 300 heures passées à scruter les Nymphéas m'ont permis d'appréhender non seulement les couleurs, les formes, mais aussi de comprendre et de ressentir la composition émanant de cet ensemble.

Huit toiles, certaines en diptyque, et une vingtaine de gouaches réalisées sur place, forment le corpus d'œuvres de cette série.

La composition m'est venue presque naturellement en « écoutant » la peinture de Monet, en lisant le paysage. L'œil se promène selon une trajectoire qu'il me faut reprendre sur la toile. La composition sous-jacente de l'œuvre de Claude Monet est devenue pour moi de plus en plus lisible. Les toiles me sont apparues très construites, pas seulement dans leur verticalité mais aussi dans les mouvements des reflets, le positionnement des nymphéas formant parfois des cercles et aussi des « damiers rythmiques ». Le regard du spectateur est emmené le long du paysage, de la toile. C'est sur cette charpente que ma composition musico-picturale peut s'inscrire.

Chaque panneau des Nymphéas a «sa » musique qui lui est proche : dans ce « Matin aux Saules », j'entrevois « La Vallée d’Obermann » de Franz Liszt par Roger Muraro ; dans « Les Deux Saules » le $3^{\mathrm{e}}$ Opus des Moments Musicaux de Franz Schuber par David Fray; etc.

Un site internet est dédié au projet de la musicalité des Nymphéas : www. waterliliespaintingmusic.com. Une vidéo présente les séances de travail au Musée de l'Orangerie : www.youtube.com/watch? $=3 \mathrm{fkq}$ TXKeZ0 